\title{
recillunds
}

Revista Científica Mundo de la Investigación y el Conocimiento

Paul Ricardo Vilatuña Fustillos a ; Elizabeth Yolanda Revelo Hidalgo b; Jessica

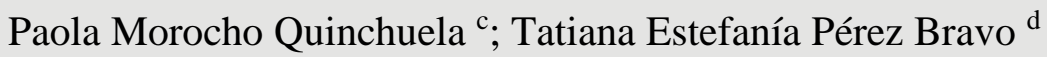

Valoración de la función renal en el embarazo

Assessment of renal function in pregnancy

Revista Científica Mundo de la Investigación y el Conocimiento. Vol. 3 núm.3, septiembre, ISSN: 2588-073X, 2019, pp. 68-81

DOI: $10.26820 /$ recimundo/3.(3).septiembre.2019.68-81

URL: http://recimundo.com/index.php/es/article/view/510

Código UNESCO: 3205 Medicina Interna

Tipo de Investigación: Artículo de Revisión

Editorial Saberes del Conocimiento

Recibido: $15 / 05 / 2019$

Aceptado: 23/06/2019

Publicado: 30/09/2019

Correspondencia: agispaulrvf@hotmail.com

a. Médico; Investigador Independiente; Guayaquil, Ecuador; agispaulrvf@hotmail.com

b. Médico General; Investigador Independiente; Guayaquil, Ecuador; elisept24@gmail.com

c. Médico General; Investigador Independiente; Guayaquil, Ecuador; jessmorocho@gmail.com

d. Médico General; Investigador Independiente; Guayaquil, Ecuador; taty_perezb@ hotmail.es 


\section{Valoración de la función renal en el embarazo}

Vol. 3, núm. 3., (2019)

Paul Ricardo Vilatuña Fustillos; Elizabeth Yolanda Revelo Hidalgo; Jessica Paola Morocho Quinchuela; Tatiana Estefanía Pérez Bravo

\section{RESUMEN}

El período de embarazo es la etapa más hermosa e importante de la mujer, no obstante, los cambios que sufre el organismo durante ella la hacen susceptible de algunas complicaciones. Una de las enfermedades más comunes que se desarrollan o se complican con el embarazo son los problemas renales. El objetivo fundamental de este estudio es plasmar los principales métodos que sirven para evaluar la función renal en la embarazada. El diseño de investigación que se llevó a cabo es de tipo documental o bibliográfico. La evaluación constante de la función renal, ya sea en pacientes sanas o con enfermedad renal previa, resulta fundamental para evitar complicaciones en la mujer embarazada y el feto. La creatinina, la ecografía renal y el comportamiento de la presión arterial, son factores resaltantes en la valoración renal de la gestante. Por último, la valoración de la función renal debe realizarse por medio de una atención interdisciplinaria conformada por obstetras y nefrólogos principalmente, y en algunos casos donde se encuentran presentes enfermedades crónicas o enfermedades renales previas, es requerida la atención de manera conjunta de endocrinólogos, cardiólogos, reumatólogos, y cualquier otra especialidad que garanticen el mejor cuidado de la paciente en gestación.

Palabras Claves: Valoración; Función Renal; Embarazo; Enfermedad. 


\title{
Valoración de la función renal en el embarazo
}

Vol. 3, núm. 3., (2019)

Paul Ricardo Vilatuña Fustillos; Elizabeth Yolanda Revelo Hidalgo; Jessica Paola Morocho Quinchuela; Tatiana Estefanía Pérez Bravo

\begin{abstract}
The pregnancy period is the most beautiful and important stage of women, however, the changes that the body undergoes during it make it susceptible to some complications. One of the most common diseases that develop or complicate with pregnancy are kidney problems. The main objective of this study is to capture the main methods used to evaluate renal function in pregnant women. The research design that was carried out is documentary or bibliographic. Constant evaluation of renal function, either in healthy patients or with previous renal disease, is essential to avoid complications in pregnant women and the fetus. Creatinine, renal ultrasound and blood pressure behavior are prominent factors in the renal assessment of the pregnant woman. Finally, the assessment of renal function must be carried out through interdisciplinary care consisting mainly of obstetricians and nephrologists, and in some cases where chronic diseases or previous renal diseases are present, joint attention by endocrinologists, cardiologists is required, rheumatologists, and any other specialty that guarantees the best care of the pregnant patient. Keywords: Assessment, Function, Renal, Pregnancy, Disease.
\end{abstract}

Key Words: Assessment; Renal Function; Pregnancy; Disease.

\section{Introducción.}




\section{Valoración de la función renal en el embarazo}

Vol. 3, núm. 3., (2019)

Paul Ricardo Vilatuña Fustillos; Elizabeth Yolanda Revelo Hidalgo; Jessica Paola Morocho Quinchuela; Tatiana Estefanía Pérez Bravo

El período de embarazo es la etapa más hermosa e importante de las mujeres, no obstante, los cambios que sufre el organismo durante ella la hacen susceptible de algunas complicaciones. Es posible que estas complicaciones se presenten debido a un problema de salud previo al embarazo o desarrollar una condición durante el mismo. Dentro de las enfermedades más comunes que se desarrollan o se complican con el embarazo se encentran los problemas renales. (Biblioteca Nacional de Medicina de los Estados Unidos, 2019)

Una mujer sana puede desarrollar diversas afecciones renales durante el periodo de gestación, que pueden ir desde las formas más leves, sin consecuencias como es el caso de la infección de vías urinarias, hasta presentar una enfermedad renal aguda que implica riesgos tanto para el feto como para la gestante; o bien puede llegar a padecer, previo al embarazo, una enfermedad crónica, ya sea renal o de otra índole, que podrían intervenir en el término natural de la gestación, ponen en riesgo la vida de la madre y la del feto, o bien podrían traer consecuencias graves a largo plazo para la mujer embarazada.

Las enfermedades renales (ER) pueden ser subdivididas en agudas o crónicas. Para Flores, Chung, Veletanga, \& Carbo (2019) La insuficiencia renal aguda "es un síndrome que se caracteriza por una disminución brusca (horas, semanas) de la función renal y posteriormente retención nitrogenada". De esta enfermedad se pueden derivar complicaciones tales como: insuficiencia cardiaca, pericarditis, arritmias, hemorragia digestiva alta, edema pulmonar, neumonía, distrés respiratorio del adulto, convulsiones, confusión, coma, entre otros. (p. 142)

Por otra parte, la enfermedad renal crónica se conoce como el síndrome que se caracteriza por una persistente variación en la estructura o la función renal, la cual se encuentra presente por 


\section{Valoración de la función renal en el embarazo}

Vol. 3, núm. 3., (2019)

Paul Ricardo Vilatuña Fustillos; Elizabeth Yolanda Revelo Hidalgo; Jessica Paola Morocho Quinchuela; Tatiana Estefanía Pérez Bravo

un periodo mayor de tres meses, y con repercusiones en la salud de la persona. Se estima que esta enfermedad afecta a cerca del $10 \%$ de la población mundial. Es prevenible sin embargo no tiene cura, generalmente es progresiva, silenciosa y se caracteriza por no presentar síntomas sino hasta etapas avanzadas, en cuyos casos el tratamiento es altamente invasivo y costoso. (Organización Panamericana de la Salud, 2015)

La Organización Mundial de la Salud (2012) en su Guía para la aplicación de la Clasificación Internacional de Enfermedades (CIE-10) a las muertes ocurridas durante el embarazo, parto y puerperio: CIE-MM describe la insuficiencia renal como una afección que constituyen causa improbable de muerte pero que puede contribuir a los eventos que la desencadenan (afección contribuyente) y que es preciso documentar esta como cualquier otra complicación que lleven a la defunción de la embarazada y/o su producto, ya que podría colaborar en el desarrollo de protocolos de tratamientos para su prevención en el futuro.

El presente estudio tiene el propósito de plasmar los principales métodos que sirven para evaluar la función renal en la embarazada y diagnosticar de forma temprana cualquier afección renal que se pueda presentar, a los fines de minimizar los riesgos que conlleva tanto para la madre como para el feto, o estando presente la enfermedad, sirva para tratar y minimizar el riesgo de complicaciones.

\section{Materiales y métodos.}




\section{Valoración de la función renal en el embarazo}

Vol. 3, núm. 3., (2019)

Paul Ricardo Vilatuña Fustillos; Elizabeth Yolanda Revelo Hidalgo; Jessica Paola Morocho Quinchuela; Tatiana Estefanía Pérez Bravo

Para desarrollar el presente estudio se procedió a identificar los documentos acordes con los objetivos de este estudio, los cuales fueron buscados en las principales bases de datos relacionadas con las ciencias médicas, de forma general y más específicamente con el área de nefrología y ginecología: MedlinePlus, Mayo Clinic, Organización Mundial de la Salud, sciELO, Dialnet, ELSEVIER, Asociación de Nefrología de España y RECIAMUC.

Dicha investigación estuvo limitada a la búsqueda y revisión sistemática de material bibliográfico, que sirvió para la síntesis de la evidencia disponible, de esta forma se puede clasificar como una investigación de tipo documental bibliográfica.

La búsqueda se realizó utilizando los descriptores y calificadores siguientes: "enfermedades renales en el embarazo", "enfermedad renal", "evaluación de la enfermedad renal en el embarazo", "valoración de la enfermedad renal en la gestación” y "ecografía renal”. El material que se obtuvo fue filtrado utilizando los criterios de relevancia, idioma español y correlación temática, La fecha de publicación se ubicó en los últimos ocho años. No se descartó el tipo de material bibliográfico.

\section{Resultados.}

Factores de riesgo

Como en la mayoría de las enfermedades, las enfermedades relacionadas con la función renal pueden estar asociadas a algunos factores de riesgo de la embarazada, los cuales conviene puntualizar ya que la detección de estas condiciones representa una de los principales elementos en la valoración de la función renal de la gestante. Existe múltiples factores de riesgo que 


\section{Valoración de la función renal en el embarazo}

Vol. 3, núm. 3., (2019)

Paul Ricardo Vilatuña Fustillos; Elizabeth Yolanda Revelo Hidalgo; Jessica Paola Morocho Quinchuela; Tatiana Estefanía Pérez Bravo

aumentan las probabilidades de que la embarazada pueda desarrollar una enfermedad renal o bien, si la presenta previo al embarazo, estas pueden complicar su condición y representan un grave peligro para la salud de la madre y el feto, e incluso para que el embarazo llegue a un feliz término. Entre las principales se encuentran los siguientes:

\section{Medicamentos}

Pérez, Roberto, Díaz, \& Tamayo (2016) refieren que existen “algunos medicamentos empleados ante complicaciones obstétricas y clínicas durante el embarazo y el puerperio que pueden provocar..." enfermedades renales, principalmente en condiciones de hipovolemia y sepsis, tales como el ácido tranexámico, aminoglucósidos, antihipertensivos, el factor VII activado recombinante, coloides y cristaloides, entre otros. (p. 839)

\section{Edad}

En todo el mundo cada vez son más las mujeres de mayor edad que deciden embarazarse, un aumento en la edad es proporcional con las probabilidades de que la misma padezca alguna enfermedad crónica previamente y se convierta en una embarazada de alto riesgo, propensa a desarrollar alguna falla del sistema renal.

\section{Enfermedades crónicas}

Aguirre Ospina \& Buitrago (2018) mencionan al respecto, que las principales condiciones clínicas que predisponen a un deterioro en el sistema renal en la gestante son: "1. PreeclampsiaEclampsia. 2. Pielonefritis aguda 3. Glomerulonefritis Aguda y nefritis intersticial Aguda. 4. 
Paul Ricardo Vilatuña Fustillos; Elizabeth Yolanda Revelo Hidalgo; Jessica Paola Morocho Quinchuela; Tatiana Estefanía Pérez Bravo

Microangiopatía trombótica. 5. Hígado graso agudo del embarazo. 6. Obstrucción del tracto urinario. 7. Falla renal aguda”. (p. 360)

Es importante la identificación y control de estas enfermedades ya que pueden desencadenar en enfermedades renales, una de las más frecuentes y peligrosas, es la preclamsia.

\section{Preclamsia - Eclampsia}

Es un tipo de complicación de la hipertensión, cuyo tratamiento farmacológico en la gestante está limitando por los efectos maternos y en el feto que se pueden producir.

Morales, y otros (2017) definen la preeclamsia como aquella donde la paciente presenta una presión arterial sistólica de 140 mmHg o más, o bien, una presión arterial diastólica de 90 mmHg o más, cuyos valores, en el caso de la gestante, deben estar confirmados por seis horas o más de diferencia. (p. 532)

Es importante mencionar en este punto, tal como lo expresan en su trabajo Abaladejo et al. (2014) que la insuficiencia renal aguda podría ser una causa, pero en otros casos es una consecuencia de la hipertensión arterial grave. (p. 7)

Valoración de la función renal

Conocer la función renal es uno de los factores claves en la prevención de las enfermedades renales en mujeres embarazadas. No obstante, las herramientas de prevención 


\section{Valoración de la función renal en el embarazo}

Vol. 3, núm. 3., (2019)

Paul Ricardo Vilatuña Fustillos; Elizabeth Yolanda Revelo Hidalgo; Jessica Paola Morocho Quinchuela; Tatiana Estefanía Pérez Bravo

incluyen una buena valoración general y una serie de exámenes relacionados con la observación y control del funcionamiento del sistema renal.

\section{Determinar el riesgo de ERC asociada a otras enfermedades crónicas}

En principio, es fundamental que se preste especial atención a los factores de riesgo que presente la gestante los cuales pueden facilitar la aparición de cualquier afección renal. Estos factores pueden ser de "exposición" tales como la sepsis, quemaduras, choque circulatorio, enfermedad crítica, drogas nefrotóxicas, cirugía cardíaca o mayor no cardíaca, agentes contrastados, entre otras. También se encuentran factores de "susceptibilidad", como lo es el caso de edad avanzada de la paciente, raza negra, deshidratación o depleción de volumen, enfermedades crónicas del corazón, hígado, pulmón o diabetes, presencia de enfermedad renal crónica previa, anemia, entre las principales.

\section{Bioquímica ácido úrico - creatinina}

"El ácido úrico es un producto final del metabolismo de las purinas que es sintetizado principalmente en hígado e intestinos, aunque también en tejidos periféricos como el músculo, endotelio y riñones". La relación que existe entre ácido úrico y enfermedad renal es muy estrecha, dado que el ácido úrico se elimina en sus 2/3 partes por medio del riñón, en tal sentido, cuando cae el filtrado glomerular, los niveles de ácido úrico aumentan. (Goicochea, 2015)

Por otra parte, la Biblioteca Nacional de Medicina de los Estados Unidos (2019) acerca de la creatinina refiere que es un examen realizado para evaluar la función de los riñones. "La creatinina es eliminada del cuerpo completamente por estos órganos. Si la función renal es 
Vol. 3, núm. 3., (2019)

Paul Ricardo Vilatuña Fustillos; Elizabeth Yolanda Revelo Hidalgo; Jessica Paola Morocho Quinchuela; Tatiana Estefanía Pérez Bravo

anormal, los niveles de creatinina en la sangre aumentarán. Esto se debe a que se elimina menos creatinina a través de la orina".

Resulta crucial determinar la creatinina en la paciente embarazada para un diagnóstico de su condición renal. Una gran mejoría en la disminución de la mortalidad materna se encuentra determinada por el empleo de clasificaciones de diagnóstico precoz de enfermedades renales, tales como el RIFLE (Risk, Injury y Failure) o el AKIN (Acute Kidney Injury Network). En estas son aplicados dos criterios básicos, por una parte "el incremento de la creatinina, Cr, (>0,3 $\mathrm{mg} / \mathrm{dl}$ en 48 horas o el incremento de la $\mathrm{Cr}$ basal> 1,5 veces, documentado o presumible) y por otra, la disminución del volumen urinario, $(<$ de $400 \mathrm{~mL} / 24 \mathrm{~h} \mathrm{o}<20 \mathrm{~mL} / \mathrm{h})$ ", los cuales permiten establecer una correlación gravedad/pronóstico. (Pérez et. al, 2016, p. 839)

El cálculo de la función renal se lleva a cabo a partir de "la creatinina sérica que establece el cálculo de la Tasa de filtrado glomerular estimado, (FGe) en ml/minuto/1.73 m2 de superficie corporal (SC), el cual se efectúa por medio de diferentes fórmulas matemáticas”. Este examen debe ser aplicado tanto a mujeres sanas como a portadoras de enfermedades crónicas, con la finalidad de determinar el grado de disfunción o no renal, especialmente en el primer trimestre. (Pérez et. al, 2016, p. 840)

Filtrado glomerular estimado (FGe)

La tasa de filtración glomerular (TFG) o Filtrado glomerular estimado (FGe) es un examen utilizado para verificar la función de los riñones. "Específicamente, brinda un cálculo 


\section{Valoración de la función renal en el embarazo}

Vol. 3, núm. 3., (2019)

Paul Ricardo Vilatuña Fustillos; Elizabeth Yolanda Revelo Hidalgo; Jessica Paola Morocho Quinchuela; Tatiana Estefanía Pérez Bravo

aproximado de la cantidad de sangre que pasa a través de los glomérulos cada minuto. Los glomérulos son los diminutos filtros en los riñones que filtran los residuos de la sangre". (Biblioteca Nacional de Medicina de los Estados Unidos, 2019)

\section{Ecografía renal}

Para Martínez et al. (2014) la importancia de la ecografía renal es que permite descartar la presencia de una patología obstructiva de la vía urinaria, además de facilitar la identificación de anormalidades estructurales las cuales podrían sugerir la presencia de daño renal. "Los quistes renales simples aislados NO son un criterio por ellos mismos de daño renal’.

\section{Comportamiento de la presión arterial}

Para las pacientes embarazadas que presentan alguna enfermedad renal, como parte del examen físico, debe realizarse la medición seriada de la presión arterial. Este estudio "será el examen más importante debido a que los valores preconcepcionales y gestacionales, poseen valor pronóstico para el deterioro de la función renal materna postparto, la mortalidad perinatal y el empeoramiento de la presión arterial en el postparto”. (Aguirre et al., 2018, p. 366)

\section{Estudio sistemático de proteinuria}

Este examen determina la presencia de proteínas en la orina. Los riñones se encargan de filtrar los desechos de la sangre, por otra parte, se encargan de retiener lo que el cuerpo necesita, 


\section{Valoración de la función renal en el embarazo}

Vol. 3, núm. 3., (2019)

Paul Ricardo Vilatuña Fustillos; Elizabeth Yolanda Revelo Hidalgo; Jessica Paola Morocho Quinchuela; Tatiana Estefanía Pérez Bravo

entre estos requerimientos se encuentran las proteínas. No obstante, cuando el organismo presenta algunas enfermedades, este deja pasar las proteínas por los filtros de los riñones, lo que ocasiona que las proteínas se encuentren presentes en la orina. (Mayo Clinic, 2018)

La hipertensión arterial gestacional, la cual por lo general aparece después de la semana 20, se acompaña de proteinuria mínima. Mientras que la preeclampsia aparece después de la semana 20 con una Proteinuria > 3 g/día o 3+ en dos muestras espontaneas de orina en las últimas 4 horas. Este control resulta de suma importancia por cuanto puede reducir la incidencia y gravedad de la preclamsia y sus consecuencias.

\section{Conclusiones.}

Todo embarazo, dada la cantidad de cambios que produce en el organismo de la madre y las limitaciones de varias terapéuticas ante la presencia de enfermedades ya sean previas o que se desarrollen durante el proceso de gestación, debe tener un control riguroso sobre la salud de la madre y del feto para conseguir un feliz término.

Las enfermedades renales resultan unas de las más frecuentes en la mujer embarazada y en sus tipos más severos se encuentran asociadas, directa o indirectamente, con las causas de mortalidad materna-fetal, por tal razón, resulta imperioso prestar especial atención en la valoración de las funciones renales ya que constituye la base para un diagnóstico precoz y un tratamiento oportuno y eficaz para garantizar en la mayor medida la buena salud tanto de la madre como de su hijo. 


\section{Valoración de la función renal en el embarazo}

Vol. 3, núm. 3., (2019)

Paul Ricardo Vilatuña Fustillos; Elizabeth Yolanda Revelo Hidalgo; Jessica Paola Morocho Quinchuela; Tatiana Estefanía Pérez Bravo

Un adecuado seguimiento y control de la función renal de la gestante es fundamental para la prevención de las complicaciones asociadas con las enfermedades renales, estas deben ir enmarcadas dentro de la identificación de los factores de riesgo. Asimismo, la valoración debe contener la bioquímica de creatinina, en el examen físico resulta de gran ayuda la ecografía renal y el control de la presión arterial.

Por último, es importante destacar una de las recomendaciones más importantes de la literatura revisada es que la valoración de la función renal debe realizarse por medio de una atención interdisciplinaria conformada por obstetras y nefrólogos principalmente, y en algunos casos donde se encuentran presentes enfermedades crónicas o enfermedades renales previas, es requerida la atención de manera conjunta de endocrinólogos, cardiólogos, reumatólogos, y cualquier otra especialidad que garanticen el mejor cuidado de la paciente en gestación.

\section{Bibliografía.}

Abaladejo, C., Sobrino, J., \& Vázquez, S. (2014). Crisis hipertensivas: seudocrisis, urgencias y emergencias. Hipertensión Riesgo Vascular, 1-11. Recuperado el 31 de JUlio de 2019, de http://www.ascarica.org/es/wp-content/uploads/2014/11/Crisis-hipertensivas.-Urgenciasemergencias-y-pseudocrisis.pdf

Aguirre Ospina, O. D., \& Buitrago, C. A. (2018). ENFERMEDAD RENAL EN LA PACIENTE OBSTETRICA. En C. A. Restrepo V, C. A. Buitrago V., J. J. Torres, \& J. Serna, Nefrología Básica 2 (pág. 14). Colombia. Recuperado el 18 de Septiembre de 2019, de http://asocolnef.com/wp-content/uploads/2018/03/Cap37.pdf

Biblioteca Nacional de Medicina de los Estados Unidos. (07 de Mayo de 2019). MedlinePlus. $\begin{array}{llllll}\text { Recuperado el } 18 \text { de } & \text { Septiembre }\end{array}$ https://medlineplus.gov/spanish/healthproblemsinpregnancy.html

Biblioteca Nacional de Medicina de los Estados Unidos. (11 de Septiembre de 2019). MedlinePlus. Recuperado el 18 de Septiembre de 2019, de https://medlineplus.gov/spanish/ency/article/003475.htm 
Vol. 3, núm. 3., (2019)

Paul Ricardo Vilatuña Fustillos; Elizabeth Yolanda Revelo Hidalgo; Jessica Paola Morocho Quinchuela; Tatiana Estefanía Pérez Bravo

Biblioteca Nacional de Medicina de los Estados Unidos. (11 de Septiembre de 2019). MedlinePlus. Recuperado el 18 de Septiembre de 2019, de https://medlineplus.gov/spanish/ency/article/007305.htm

Flores, G. A., Chung, J. R., Veletanga, A. V., \& Carbo, J. A. (2019). Riesgo-Beneficio en el uso del catéter doble $\mathrm{J}$ en pacientes con insuficiencia renal. RECIAMUC, 3(3), 137-152. Recuperado el 17 de Septiembre de 2019, de http://reciamuc.com/index.php/RECIAMUC/article/view/271/287

Goicochea, M. (2015). Ácido úrico y enfermedad renal crónica old. (E. España, Ed.) Nefrología al día. Recuperado el 15 de Septiembre de 2019, de https://www.nefrologiaaldia.org/esarticulo-acido-urico-enfermedad-renal-cronica-18

Martínez, A., Górriz, J. L., Bover, J., Segura, J., Cebollada, J., Escalada, J., . . . Mazón, P. (2014). Documento de consenso para la detección y manejo de la enfermedad renal crónica. Nefrología, 34(2), 243-262. Recuperado el 18 de Septiembre de 2019, de https://www.revistanefrologia.com/es-pdf-X0211699514053919

Mayo Clinic. (26 de Enero de 2018). Mayo Clinic. Recuperado el 18 de Septiembre de 2019, de https://www.mayoclinic.org/es-es/symptoms/protein-in-urine/basics/causes/sym20050656

Morales, V., Reyna, E., Mejia, J., Santos, J., Torres, D., Reyna, N., \& Fernández, A. (2017). Labetalol o alfa-metildopa oral en el tratamiento dela hipertensión severa en preeclámpticas. Rev Peru Ginecol Obstet, 63(4), 529-535. Recuperado el 01 de Agosto de 2019, de http://www.scielo.org.pe/pdf/rgo/v63n4/a03v63n4.pdf

Organización Mundial de la Salud. (2012). Guía de la OMS para la aplicación de la CIE-10 a las muertes ocurridas durante el embarazo, parto y puerperio: CIE MM. Ginebra: Organización Mundial de la Salud. Recuperado el 16 de Septiembre de 2019, de https://www.paho.org/clap/index.php?option=com_docman\&view=download\&category_ slug=sip\&alias=308-guia-de-la-oms-para-la-aplicacion-de-la-cie10-a-las-muertesocurridas-durante-el-embarazo-parto-y-puerperio-cie-mm-5\&Itemid=219\&lang=es

Organización Panamericana de la Salud. (10 de Marzo de 2015). paho.org. Recuperado el 15 de Septiembre de 2019, de https://www.paho.org/hq/index.php?option=com_content\&view=article\&id=10542:2015opsoms-sociedad-latinoamericana-nefrologia-enfermedad-renal-mejorartratamiento\&Itemid $=1926 \&$ lang $=$ es

Pérez, J., Roberto, C., Díaz, J. O., \& Tamayo, R. (2016). Diagnóstico, Evaluación y Manejo de la Enfermedad Renal en el Embarazo. Revista Habanera de Ciencias Médicas, 15(5), 834858. Recuperado el 18 de Septiembre de 2019, de https://pdfs.semanticscholar.org/4e94/e7fd93fd6eb59d76c4f23d859fa35b5485b1.pdf 